\title{
HDAC inhibitor LMK-235 promotes the odontoblast differentiation of dental pulp cells
}

\author{
ZHAO LIU ${ }^{1}$, TING CHEN ${ }^{1}$, QIANQIAN HAN ${ }^{2}$, MING CHEN $^{1}$, \\ JIE YOU ${ }^{1}$, FUCHUN FANG ${ }^{1}$, LING PENG ${ }^{1}$ and BULING WU ${ }^{1}$ \\ ${ }^{1}$ Department of Stomatology, Nanfang Hospital, Southern Medical University, Guangzhou, Guangdong 510515; \\ ${ }^{2}$ Department of Periodontics, Stomatology Hospital of Guangdong Province, Guangzhou, Guangdong 510260, P.R. China
}

Received January 6, 2017; Accepted August 18, 2017

DOI: $10.3892 / \mathrm{mmr} .2017 .8055$

\begin{abstract}
The role of dental pulp cells (DPCs) in hard dental tissue regeneration had received increasing attention because DPCs can differentiate into odontoblasts and other tissue-specific cells. In recent years, epigenetic modifications had been identified to serve an important role in cell differentiation, and histone deacetylase (HDAC) inhibitors have been widely studied by many researchers. However, the effects of HDAC4 and HDAC5 on the differentiation of DPCs and the precise molecular mechanisms remain unclear. The present study demonstrated that LMK-235, a specific human HDAC4 and HDAC5 inhibitor, increased the expression of specific odontoblastic gene expression levels detected by reverse transcription-quantitative polymerase chain reaction (RT-qPCR) in dental pulp cells, and did not reduce cell proliferation tested by MTT assay after 3 days in culture at a low concentration. In addition, the mRNA and protein expression levels of dentin sialophosphoprotein, runt-related transcription factor 2, alkaline phosphatase (ALP) and osteocalcin were evaluated by RT-qPCR and western blotting, respectively. The increased gene and protein expression of specific markers demonstrated, indicating that LMK-235 promoted the odontoblast induction of DPCs. ALP activity and mineralised nodule formation were also enhanced due to the effect of LMK-235, detected by an ALP activity test and Alizarin Red S staining, respectively. Additionally, the vascular endothelial growth factor (VEGF)/RAC-gamma serine/threonine-protein kinase $(\mathrm{AKT}) / \mathrm{mechanistic}$ target of rapamycin (mTOR) signalling pathway was tested to see if it takes part in the differentiation of DPCs treated with LMK-235, and it was demonstrated
\end{abstract}

Correspondence to: Professor Buling $\mathrm{Wu}$ or Ting Chen, Department of Stomatology, Nanfang Hospital, Southern Medical University, 1838 Guangzhou Avenue North, Guangzhou, Guangdong 510515, P.R.China

E-mail: bulingwu1958@126.com

E-mail:252199453@qq.com

Key words: LMK-235, dental pulp cells, odontoblast differentiation, dentin sialophosphoprotein, histone deacetylase 4 that the mRNA expression levels of VEGF, AKT and mTOR were upregulated. These findings indicated that LMK-235 may serve a key role in the proliferation and odontoblast differentiation of DPCs, and could be used to accelerate dental tissue regeneration.

\section{Introduction}

Human dental mesenchymal stem cells (hDMSCs) can be isolated from various types of dental tissues, such as periodontal ligament, dental pulp, alveolar bone marrow and periapical follicle, and have the ability to differentiate into different cell types, including odontoblasts, osteoblasts, adipocytes and chondrocytes, with the purpose of cell based tissue engineering $(1,2)$. Previous studies have used hDMSCs for dental tissue regeneration (3). Dental pulp cells (DPCs) can be obtained from extracted third molar teeth, which are clinically regarded as 'waste tissue', and can differentiate into odontoblasts that serve as the precursor cells for dentin formation. However, the precise mechanism of DPCs odontoblast differentiation is still unclear.

In recent years, epigenetic regulation had been proven to serve an important role in the differentiation of many cells. Epigenetic modification of chromatin does not result in changes to the DNA sequence, but instead modifies the structure and the accessibility of chromatin, leading to heritable alterations of gene expression during cell division (4). Epigenetic modification of gene expression explicitly operates through chromatin remodelling, such as nucleosome structural modification. Acetylation and deacetylation controlled by histone acetyltransferases (HATs) and histone deacetylases (HDACs) are the common methods of post-translational histone modification (5). HDAC inhibitors (HDACi) have been widely used in oncotherapy due to the potent effects of inducing cell cycle arrest, apoptosis and differentiation of tumor cell lines (6). Furthermore, it had been demonstrated that HDACi can promote osteoblastic differentiation and bone formation by increasing the expression of osteogenic-associated proteins, including alkaline phosphatase (ALP), osteocalcin (OCN), osterix and runt-related transcription factor 2 (Runx2), and accelerating mineralization via HDAC inhibition $(7,8)$. Class I and class II HDACs have been demonstrated to be highly expressed in human periodontal ligaments, while HDAC3 expression is decreased gradually during 
osteogenic differentiation. Meanwhile, inhibition of HDAC3 by trichostatin A (TSA) might accelerate osteoblast differentiation in periodontal ligament cells through hyperacetylation of Histone H3 (9). Osteoblast differentiation could also be stimulated by HDAC1 inhibition via the downregulation of osterix and OCN (10). In contrast, long-term use of VPA, an HDAC inhibitor thatis also used as an antiepileptic drug, reduces bone mineral density as well as content in vivo, which is possibly due to the systemic use of VPA and a relatively high dose (11). Therefore, the appropriate usage and dosage of HDACi for activating cell differentiation needs to be determined before use.

Previous studies regarding HDAC expression in human dental tissue, especially dental pulp, indicated that HDAC1, 2, 3, 4 and 5 were highly expressed in odontoblasts, and class IHDACi has been demonstrated to promote differentiation and increase mineralization at relatively low concentrations $(12,13)$. Kwon et al (14) demonstrated that HDAC inhibition enhanced odontoblast differentiation and increased dentin sialophosphoprotein (DSPP) expression in odontoblast-like cells partially by increasing the expression of nuclear factor $1 \mathrm{C}$-type. Class IIHDACs such as HDAC4 and HDAC5 are closely associated with osteoblast differentiation. It was demonstrated that acetylation of osteoblast-specific transcription factor osterix (Osx) was essential for osteoblast differentiation of $\mathrm{C} 2 \mathrm{C} 12$ cells, while deacetylation of Osx mediated by HDAC4 may serve the opposite role (15). Through inhibiting HDAC5 during osteogenic differentiation of vascular smooth muscle cells, microRNA-2861 could upregulate Runx2 protein expression levels (16). However, the effects of HDAC4, HDAC5and their specific inhibitor on odontoblast differentiation of DPCs, and the precise molecular and epigenetic mechanisms behind this process, remain unclear. LMK-235 is a human specific HDAC4 and HDAC5 inhibitor $(17,18)$, and the present study aimed to investigate how LMK-235 affects the proliferation and differentiation of DPCs in vitro, and identify its possible molecular mechanism. This study may provide novel ideas for odontoblast differentiation of dental pulp derived cells and dentin regeneration.

\section{Materials and methods}

Primary cell culture. Human third molars were collected from healthy young men (18-25 years of age) at the Department of Oral and Maxillofacial Surgery, Nanfang Hospital, Southern Medical University (Guangzhou, China). This study was approved by the Institutional Review Board at the Department of Stomatology, Nanfang Hospital, Southern Medical University. Written informed consent was obtained from the patients prior to the study. The pulp tissue was gently separated from the extracted third molars and subsequently digested in a solution of $3 \mathrm{mg} / \mathrm{ml}$ collagenase type I (Sigma-Aldrich; Merck KGaA, Darmstadt, Germany) at $37^{\circ} \mathrm{C}$ for $30 \mathrm{~min}$. The pulp tissue was cultured in $10 \%$ fetal calf serum with $100 \mathrm{U} / \mathrm{ml}$ penicillin and $100 \mathrm{mg} / \mathrm{ml}$ streptomycin (all from Hyclone, Logan, UT, USA) and cultured at $37^{\circ} \mathrm{C}$ in $5 \% \mathrm{CO}_{2}$. The medium was changed $24 \mathrm{~h}$ later and then changed every 3 days. The primary cells were used at passage 3-5 in this study.

Cell treatment. To evaluate the appropriate concentration of LMK-235 (Selleck Chemicals, Houston, TX, USA) to induce
DPC odontoblast differentiation without cell proliferation reducing, cells were treated with different concentrations $(0,50,100,250,500$ and 1,000 nM) of LMK-235 for 3 days, and the $0 \mathrm{nM}$ group was set as the control group. The gene expression levels of odontoblast markers, such as DSPP, ALP and Runx2, were detected by reverse transcription-quantitative polymerase chain reaction (RT-qPCR).

After the concentration was confirmed, the effect of LMK-235 was tested onodontoblast differentiationin normal medium [Dulbecco's modified Eagle's medium supplemented with $10 \%$ fetal bovine serum (Hyclone)] and mineralizing medium. Cells $\left(1 \times 10^{6}\right)$ were seeded in a $60-\mathrm{mm}$ culture dish, and the cells were divided into the following four groups: Control (incubated in normal growth medium and Dulbecco's modified Eagle's medium supplemented with $10 \%$ fetal bovine serum), LMK-235 (in normal growth medium supplemented with $100 \mathrm{nM}$ LMK-235), mineralized inductive (MI; in mineralizing inductive medium), and LMK-235 + mineralized inductive (MI+LMK-235; in mineralizing inductive mediumsupplemented with $100 \mathrm{nM} \mathrm{LMK-235).} \mathrm{The}$ mineralizing inductive medium contained $10 \%$ fetal bovine serum, $50 \mathrm{mg} / \mathrm{ml}$ ascorbic acid, $10 \mathrm{mM} \beta$-glycerophosphate and $100 \mathrm{nM}$ dexamethasone (all from Sigma-Aldrich; Merck KGaA). The medium was changed every 3 days. ALP activity was tested 1week after treatment, and Alizarin Red S staining (room temperature, $30 \mathrm{~min}$ ) was completed after a 21-day treatment. Furthermore, the mRNA and protein expression levels of DSPP, ALP, Runx 2 and OCN were detected by RT-qPCR and western blotting after 7, 14 and 21 days of culture. To explore the potential mechanism behind odontoblast differentiation activated by LMK-235, the mRNA expression levels of vascular endothelial growth factor (VEGF), RAC-gamma serine/threonine-protein kinase (AKT3) and mechanistic target of rapamycin (mTOR) were detected soon afterwards.

Cell proliferation assay. To evaluate the effects of LMK-235 on cell viability at different concentrations, cell proliferation and viability were measured using MTT assay. Briefly, DPCs $\left(4.0 \times 10^{3}\right.$ cells/well) were seeded into 96-well plates and incubated for $24 \mathrm{~h}$. Various concentrations of LMK-235 were added $(0,50,100,250,500$ and $1,000 \mathrm{nM})$ in a culture medium volume of $100 \mathrm{ml}$ in each well. MTT $(\sim 15 \mu \mathrm{l} ; 5 \%$; Sigma-Aldrich; Merck KGaA) was added at the1, 3, 5 and 7-day time points. The DPCs were further incubated for $4 \mathrm{~h}$ in $5 \% \mathrm{CO}_{2}$ at $37^{\circ} \mathrm{C}$, and then $100 \mathrm{ml}$ dimethyl sulfoxide (Sigma-Aldrich; Merck $\mathrm{KGaA}$ ) was added to dissolve the formazan product. Each condition was prepared 5 times, and the optical densities were read using an enzyme-linked immunosorbent assay reader at $562 \mathrm{~nm}$.

RNA preparation and RT-qPCR. Total RNA extracted from DPCs was prepared using RNAiso Plus (Takara Biotechnology Co., Ltd., Dalian, China) according to the manufacturer's protocol. cDNA was synthesized from $0.5 \mathrm{mg}$ total RNA using the PrimeScript RT Reagent kit (Takara Biotechnology Co., Ltd.). qPCR was completed using SYBR Green PCR Master Mix (Takara Biotechnology Co., Ltd.) on an ABI Prism 7500 sequence detection system. The reaction conditions were according to the manufacturer's protocol as follows: 40 cycles of denaturation at $95^{\circ} \mathrm{C}$ for $15 \mathrm{sec}$ and amplification at $60^{\circ} \mathrm{C}$ 
for $60 \mathrm{sec}$. All reactions of each sample were tested in triplicate and normalized to $\beta$-actin Mrna (19). All of the specific primers are listed in Table I.

Western blot analysis. DPCs were solubilized in lysis buffer (Beyotime Institute of Biotechnology, Haimen, China) for $20 \mathrm{~min}$, followed by centrifugation at $4^{\circ} \mathrm{C}$ at $14,000 \mathrm{x} \mathrm{g}$ for $20 \mathrm{~min}$. The protein concentrations of the cell lysates were detected using a Protein Assay kit (Beyotime Institute of Biotechnology). Bromophenol blue (10\%) was added to $20 \mu \mathrm{g}$ protein in each group, the mixture was boiled for $5 \mathrm{~min}$ and $10 \%$ SDS-PAGE was used to separate the total protein. After electrophoresis, proteins in the gels were transferred to polyvinylidene fluoride membranes (EMD Millipore, Billerica, MA, USA). The membranes were blocked afterwards in 5\% low-fat milk solubilized in Tris-buffered saline with 0.1 Tween-20 (TBST) at room temperature for $1 \mathrm{~h}$, and rinsedwithTBST three times (10 min each). The membranes then were incubated with primary rabbit against human monoclonal antibodies: GAPDH (1:10,000; cat no. ab128915) and Runx2 (1:1,000; cat no. ab192256) (both from Abcam, Cambridge, MA, USA), and mouse anti-human DSPP (1:1,000; cat no. sc-73632; Santa Cruz Biotechnology, Inc., Dallas, TX, USA), overnight at $4^{\circ} \mathrm{C}$. After three washes with TBST (10 min each), the membranes were incubated again with goat anti-mouse IgG (1:10,000; cat no. 926-32210) or goat anti-rabbit IgG (1:10,000; cat no. 926-68021) (both from LI-COR Biosciences, Lincoln, NE, USA) secondary antibodies for $1 \mathrm{~h}$ at room temperature. Membranes were then washed three times with TBST, and then visualized using an Odyssey infrared imaging system (LI-COR Biosciences). Integrated optical density was detected using ImageJ version 1.47 (National Institutes of Health, Bethesda, MD, USA).

ALP activity test. For the ALP activity test, cells cultured for 1 week were fixed with $10 \%$ formalin, incubated with $0.1 \%$ Triton X-100 for $5 \mathrm{~min}$ at room temperature, and then the ALP activity was determined using the Leukocyte Alkaline Phosphatase kit (Sigma-Aldrich; Merck KGaA) according to the manufacturer's protocol. The absorbance was detected using a microplate reader at a wavelength of $520 \mathrm{~nm}$. A Bicinchoninic Acid protein assay kit (Beyotime Institute of Biotechnology) was used to quantify the protein content. The ALP activities in the DPCs of the 4 groups were normalized against the total protein content.

Alizarin Red $S$ staining. Cells were cultured in 6-well plates for 21 days, washed using phosphate-buffered saline (PBS) and then fixed with $4 \%$ formalin for $10 \mathrm{~min}$. The cells were stained with 2\% Alizarin Red S (Sigma-Aldrich; Merck $\mathrm{KGaA}$ ) dissolved in distilled water, $\mathrm{pH} 4.4$, at room temperature for $30 \mathrm{~min}$. The cells were gently washed with distilled water three times to remove the unbound Alizarin Red S. The stained cells were imaged using an inverted microscope.

Statistical analysis. Statistical analysis were performed using SPSS 19.0 software (IBM Corp., Armonk, NY, USA). Data are expressed as the mean \pm standard deviation. Differences were analysed using a one-way analysis of variance followed by a Bonferroni test. $\mathrm{P}<0.05$ was considered to indicate a statistically significant difference.
Table I. Primers used for reverse transcription-quantitative polymerase chain reaction.

\begin{tabular}{ll}
\hline Gene & \multicolumn{1}{c}{ Sequence } \\
\hline 3-actin & F: 5'-GAGCTACGAGCTGCCTGACG -3' \\
& R: 5'-CCTAGAAGCATTTGCGGTGG -3' \\
DSPP & F: 5'-TGGCGATGCAGGTCACAAT -3' \\
& R: 5'-CCATTCCCACTAGGACTCCCA -3' \\
Runx2 & F: 5'-TCAACGATCTGAGATTTGTGGG -3' \\
& R: 5'-GGGGAGGATTTGTGAAGACGG -3' \\
ALP & F: 5'-CCAAAGGCTTCTTCTTGCTG-3' \\
& R: 5'-CCACCAAATGTGAAGACGTG-3' \\
OCN & F: 5'-GGCGCTACCTGTATCAATGG -3' \\
& R: 5'-GTGGTCAGCCAACTCGTCA -3' \\
VEGF & F: 5'-CTACCTCCACCATGCCAAGT-3' \\
& R: 5'-CACACAGGATGGCTTGAAGA-3'
\end{tabular}

AKT3

mTOR F: 5'-AATGGACAGAAGCTATCCAGGC -3' R: 5'-TGATGGGTTGTAGAGGCATCC -3'

F: 5'-TCCGAGAGATGAGTCAAGAGG -3' R: 5'-CACCTTCCACTCCTATGAGGC -3'

F, forward; R, reverse; DSPP, dentin sialophosphoprotein; Runx2, runt-related transcription factor 2; ALP, OCN, osteocalcin; VEGF, vascular endothelial growth factor; AKT3, RAC-gamma serine/threonine-protein kinase; mTOR, mechanistic target of rapamycin.

\section{Results}

Effect of LMK-235 on cell proliferation. HDACi might induce growth arrest and apoptosis in tumor cells and non-tumor cells $(20,21)$. The results of the present study indicated that cell growth was reduced in the 250 and $500 \mathrm{nM}$ groups compared with the $0 \mathrm{nM}$ group at days 3 and 5. In addition, the proliferation of the 1,000 $\mathrm{nM}$ group was reduced at days 1,5 and 7 compared with the $0 \mathrm{nM}$ group. However, low concentrations of LMK-235 (50 and $100 \mathrm{nM}$ ) barely affected the proliferation of DPCs (Fig. 1A).

Effect of LMK-235 on ALP activity in DPCs. The LMK-235 treated group demonstrated no significant differences in ALP activity compared with the control group. However, ALP activity of the MI+LMK-235 group was significantly increased when compared with the MI group (Fig. 1B).

Determination of LMK-235 concentration to promote odontoblast differentiation. To find the proper concentration of LMK-235 to promote DPCs odontoblast differentiation, the expression levels of odontoblast-specific genes were detected in DPCs incubated with different concentrations of LMK-235 for 3 days by RT-qPCR. The expression of ALP mRNA was significantly upregulated at low concentrations of LMK-235 (50 and $100 \mathrm{nM}$ ) when compared with the control group (Fig. 1C). In addition, the expression of Runx2 was higher in the 50,100 and $250 \mathrm{nM}$ groups than in the control group, although these differences were not statistically 


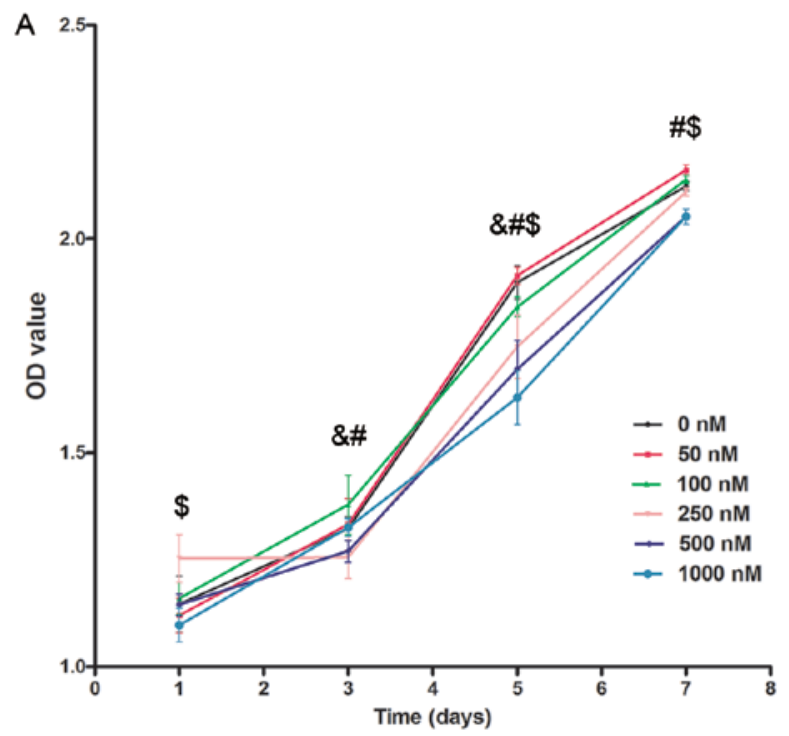

C

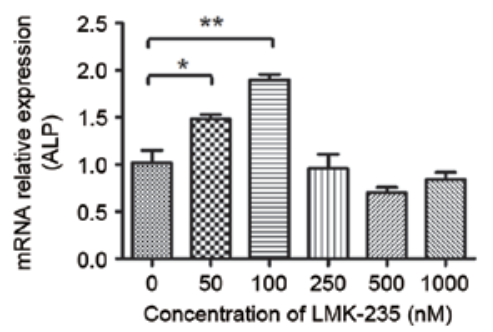

B

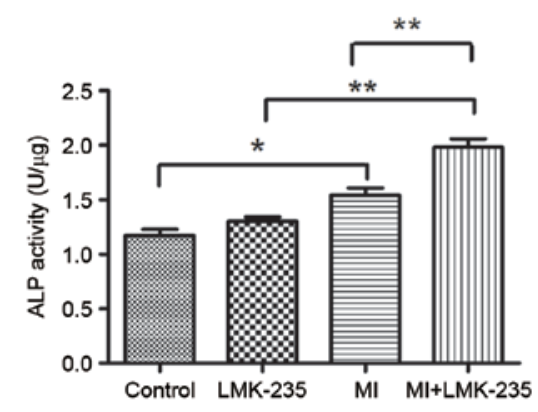

Figure 1. Effect of LMK-235 on DPCs. (A) Cell viability, OD value for $0 \mathrm{nM}$ group was compared with the remaining groups. ${ }^{\circledR} \mathrm{P}<0.05 \mathrm{vs} .250 \mathrm{nM}$; ${ }^{\#} \mathrm{P}<0.05 \mathrm{vs}$. $500 \mathrm{nM} ;{ }^{\$} \mathrm{P}<0.05$ vs. $1,000 \mathrm{nM}$. (B) ALP activity, and (C) ALP, (D) Runx2 and (E) DSPP mRNA expression levels in DPCs following treatment with LMK-235. ${ }^{*} \mathrm{P}<0.05,{ }^{* *} \mathrm{P}<0.01,{ }^{* * *} \mathrm{P}<0.001$. Data are presented as the mean \pm standard deviation. OD, optical density; DPCs, dental pulp cells; ALP, alkaline phosphatase; DSPP, dentin sialophosphoprotein; Runx2, runt-related transcription factor 2; MI, mineralized inductive.

significant (Fig. 1D). Furthermore, the expression of DSPP mRNA was also increased in the DPCs of the 50,100 and $250 \mathrm{nM}$ groups compared with the control group (Fig. 1E). The expression level of the $100 \mathrm{nM}$ group was 8.22-times that of the control group $(\mathrm{P}<0.001)$. Conversely, the expression levels of all three of these factors were downregulated in the 500 and $1,000 \mathrm{nM}$ groups.

Combined with the effect of LMK-235 on cell proliferation, $100 \mathrm{nM}$ was chosen as the experimental concentration at which cell proliferation was not affected, and the expressions of odontoblast genes were upregulated in DPCs.

LMK-235 combined with mineralizing medium increases the $m R N A$ expression of odontoblast-specific factors in DPCs. As presented in Fig. 2, the effect of LMK-235 treatment on DPCs during mineralized induction was evaluated. DSPP and ALP mRNA expression levels were increased in the MI+LMK-235 group and the MI group compared with the LMK-235 treated group and the control group at days 7 and 14 respectively. Furthermore, them RNAexpression levels in the MI+LMK-235 group were significantly higher than that in the MI group at those times. At day 21, there was no significant differences in DSPP and ALP mRNA expression between the MI+LMK-235 and the MI groups (Fig. 2A and B, respectively).

Runx 2 mRNA expression was significantly increased in the MI+LMK-235 and MI groups when compared with the
LMK-235 treated group and the control group at days 7 and 14. The expression levels in the MI+LMK-235 group and MI group were 1.45- and 1.47-times those in the LMK-235 group and the control group, respectively, with a significant difference at day 21. In addition, the Runx 2 mRNA expression level in the MI+LMK-235 group was notably increased relative to that of the MI group at day 21 (Fig. 2C).

OCN mRNA expression was not altered significantly among the 4 groups at days 7 and 14 . However, OCN mRNA expression in the MI+LMK-235 group and the MI group was 1.51- and 1.45-timesthat of the LMK-235 treated group and the control group, respectively, at day 21 , whereas there was no significant difference between the expression levels in the MI+LMK-235 and MI groups (Fig. 2D).

LMK-235 combined with mineralizing medium increases the protein expression of odontoblast specific factors in DPCs. As presented in Fig. 3, DSPP protein expression was markedly higher in the MI+LMK-235 group compared with the MI group at days 7 and 14. At day 21, DSPP protein expression was slightly higher in the MI+LMK-235 group compared with the MI group without significant difference. Runx2 protein expression was upregulated by LMK-235 treatment in normal culture medium and mineralizing medium, and the expression level in the MI+LMK-235 group was 1.22-, 1.29- and 1.17-times that in the MI group at days 7,14 and 21, respectively (Fig. 3). 
A

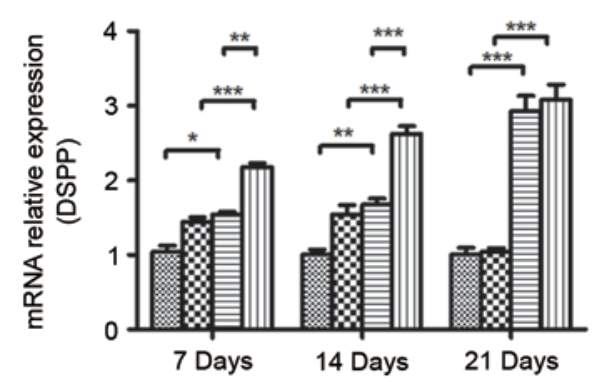

C
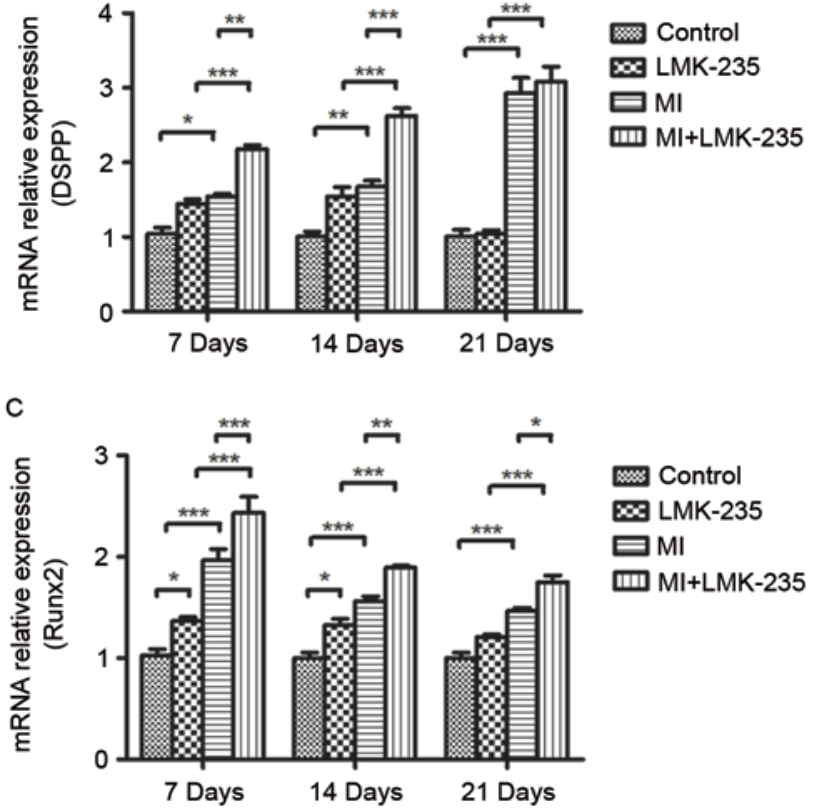

B

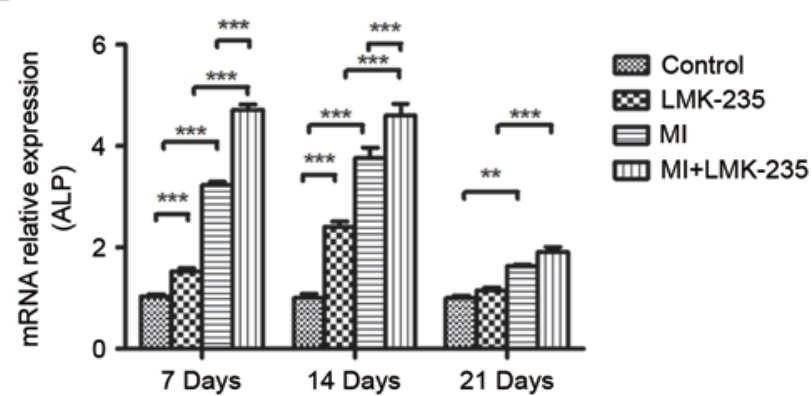

D

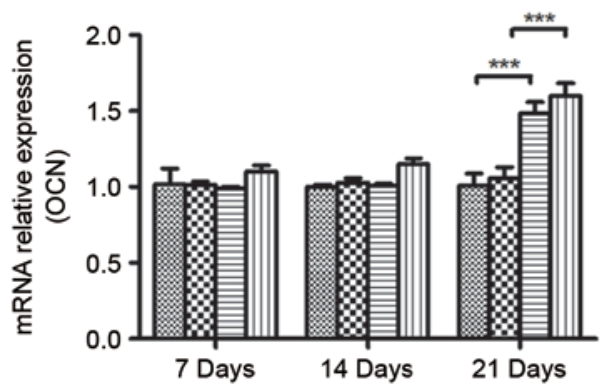

网 Control $\infty$ LMK-235 曰MI 四 Ml+LMK-235

Figure 2. LMK-235 combined with mineralizing medium increases the mRNA expression of odontoblast-specific factors in DPCs. mRNA expression levels of (A) DSPP, (B) ALP, (C) Runx2 and (D) OCN in DPCs following treatment with LMK-235 for 7, 14 and 21 days. Data are presented as the mean \pm standard deviation. ${ }^{*} \mathrm{P}<0.05,{ }^{* *} \mathrm{P}<0.01,{ }^{* * *} \mathrm{P}<0.001$. DPCs, dental pulp cells; ALP, alkaline phosphatase; DSPP, dentin sialophosphoprotein; Runx2, runt-related transcription factor 2; MI, mineralized inductive; OCN, osteocalcin.

Effect of LMK-235 on Alizarin Red S staining of DPCs. The control group and the LMK-235 treated group exhibited limited mineralized nodules stained by Alizarin Red S. Conversely, the MI+LMK-235 group exhibited more and larger calcified nodules and stronger staining when compared with the MI group (Fig. 4A-D).

VEGF/AKT/mTOR pathway may take part in odontoblast differentiation induced by $L M K-235$. mRNA expression of VEGF and AKT3 in the MI+LMK-235 group was increased significantly compared with the MI group at days 7 and 14. At day 21, the mRNA expression of AKT3 was notably upregulated in the MI+LMK-235 group compared with that of the MI group, whereas there was no significant difference between the mRNA levels of VEGF in the MI+LMK-235 and the MI groups (Fig. 4E and F). mTOR mRNA expression increased slightly with no significant difference in the MI+LMK-235 group compared with the MI group at days 7, 14 and 21 (Fig. 4G).

\section{Discussion}

The present study focused on the potential molecular mechanism underlying the role of LMK-235 in odontoblast differentiation of DPCs, which possibly would influence dentin formation and regeneration process. In this experiment, the proliferative potential of DPCs cultured with different concentration of LMK-235 was investigated. High concentrations of LMK-235 decreased the proliferation of DPCs compared with that of the control group, whereas the proliferation of those DPCs treated with low concentrations of LMK-235 (50 and $100 \mathrm{nM}$ ) was not noticeably affected. These results were consistent with Paino et al (12), who demonstrated that low concentrations of VPA did not reduce cell viability of either DPSCs or primary osteoblasts after 2 days of culture, where as treatment with high concentrations of VPA for $>48$ h decreased cell proliferation. To confirm the appropriate concentration of LMK-235 that might promote DPC odontoblast differentiation, the gene expression levels of specific factors during odontoblast differentiation were determined by RT-qPCR. The results indicated that DSPP, ALP and Runx2 mRNA expression levels, which were of great importance in odontoblast differentiation (13), were increased in DPCs when cultured with a low concentration of LMK-235, especially at the concentration of $100 \mathrm{nM}$. In this sense, the concentration of 100 nMLMK-235 activated the odontoblast differential potency of DPCs without reducing cell proliferation in vitro. According to the results mentioned above, LMK-235 at a concentration of $100 \mathrm{nM}$ was subsequently used to study DPC odontoblast differentiation.

ALP is an important marker in the early phase of odontoblast differentiation, and its activity has been reported to markedly increase during this process (22). The ALP activity in this experiment indicated that LMK-235 might exhibit a more obvious effect on enhancing ALP activity of DPCs with the synergistic fact of mineralizing medium. The mRNA expression of ALP was increased markedly in the MI+LMK-235 group compared with the MI group at days 7 and 14, indicating hat LMK-235 might increase ALP expression during the early stages of odontoblast differentiation. Changes between ALP gene expression and activity in the LMK-235 treated group and the control group were inconsistent, and this might be caused by delayed protein expression. There was no significant difference in ALP mRNA expression between the MI+LMK-235 and MI groups at days 21 . This effect might be due to that LMK-235 accelerated DPC odontoblast differentiation and ALP expression reached a peak in the early stage; thus, there 
A

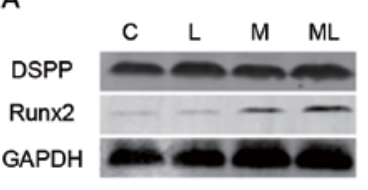

B

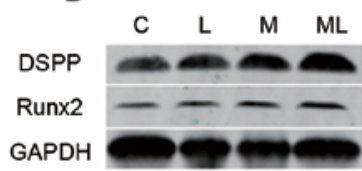

C

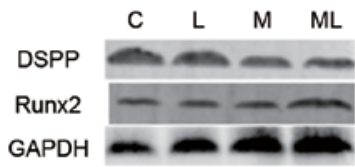

D

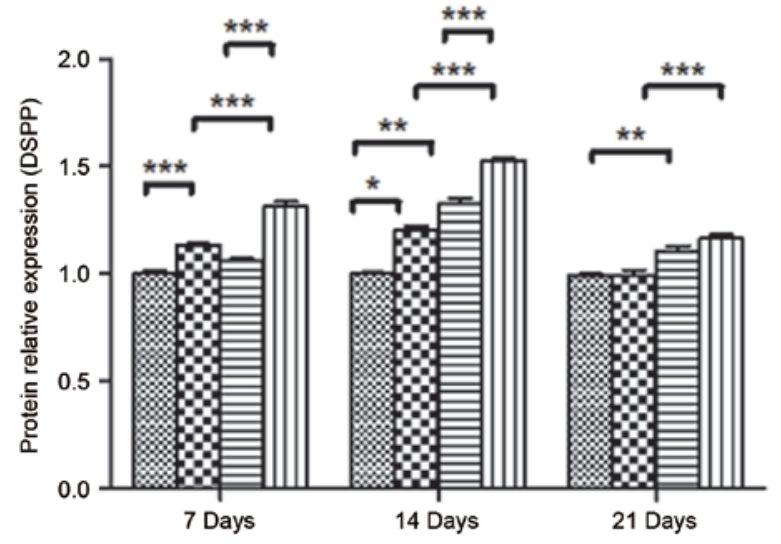

Control

Q

哣

एणा MI+LMK-235

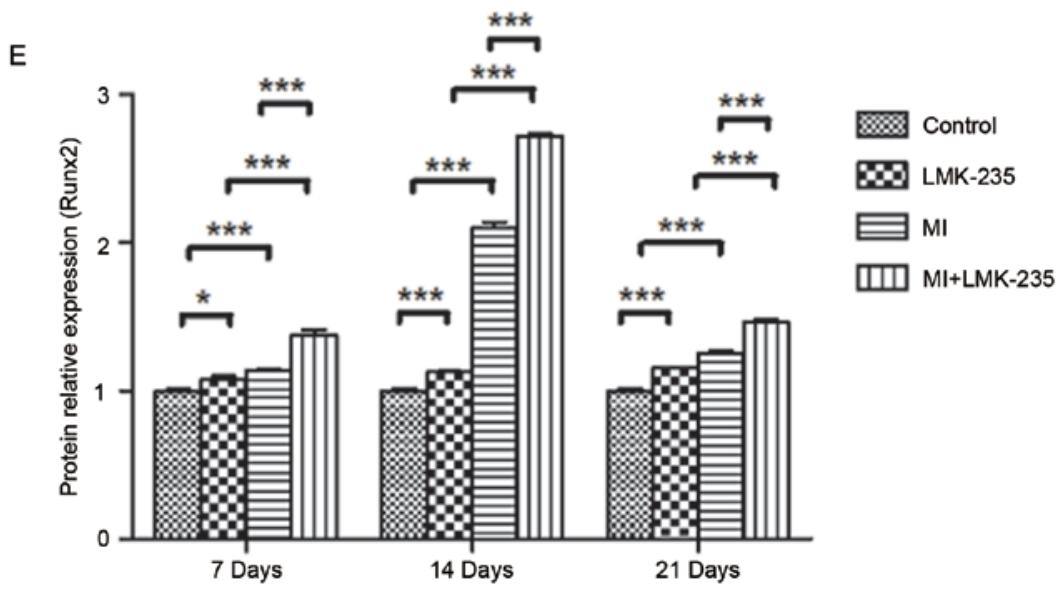

Figure 3. LMK-235 combined with mineralizing medium increases the protein expression of odontoblast specific factors in DPCs. Representative western blot images of protein expression levels of DSPP and Runx2 at (A) 7, (B) 14 and (C) 21 days. C, control group; L, LMK-235 group; M, MI group; ML, MI+LMK-235 group. Quantification of (D) DSPP and (E) Runx2 protein expression levels. Data are presented as the mean \pm standard deviation. ${ }^{*} \mathrm{P}<0.05,{ }^{* * *} \mathrm{P}<0.01,{ }^{* * * *} \mathrm{P}<0.001$. DPCs, dental pulp cells; DSPP, dentin sialophosphoprotein; Runx2, runt-related transcription factor 2; MI, mineralized inductive.

were no significant differences that could be identified between the MI+LMK-235 and MI groups at later stage.

DSPP, a member of small integrin-binding ligand N-linked glycoproteins, is widely regarded as a specific marker of odontoblast that regulates the progress of dentin formation, and its protein is nearly 400x more expressed in the dentin than in the bone (23). DSPP is reported to be overexpressed at 1-3 days after injury in vivo and upregulated during the early stages of odontoblast differentiation in vitro (24). DSPP expression was proven to be regulated by histone modification. Gu et al (25) demonstrated that after DPSC were cultured in osteo differentiation medium, the increase of DSPP expression was associated with histone $\mathrm{H} 3$ acylation. Wang et al (26) indicated that p300, a HAT, could promote gene expression of odontoblast markers via enhancing acetylation of $\mathrm{H} 3 \mathrm{~K} 9$ in the promoter regions of DSPP gene. In the present study, RT-qPCR analysis demonstrated that DSPP mRNA expression in the MI+LMK-235 group was significantly upregulated when compared with that of the MI group at days 7 and 14, and was slightly higher at day 21. Protein expression examined by western blotting confirmed the outcomes above. These results indicated that LMK-235 may increase the expression of DSPP, especially during the early stages of differentiation, which contributed to DPC odontoblast differentiation.

Runx2, a crucial factor of odontoblast and osteoblast differentiation at both the early and late stages, is reported to be continuously upregulated during the differentiation of mesenchymal stem cells, especially at the late period (27). The results of Runx 2 expression was not consistent as those by Jin et al (13) that Runx2 expression was not affected in DPSCs treated with TSA. The reason behind these differences might be that HDAC4 could modulate Runx2 activity (28), and Runx 2 gene and protein can be upregulated due to the inhibition of HDAC4 by LMK-235. These data above suggested that LMK-235 might improve the expression of Runx 2 during odontoblast differentiation. The expression of DSPP is elevated in 3-day-old transgenic mice overexpressing Runx2, but decreased in those mice by the age of 1 month (29). 


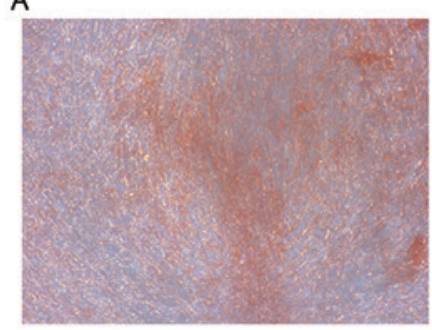

B

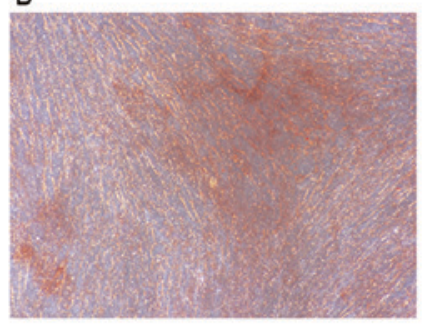

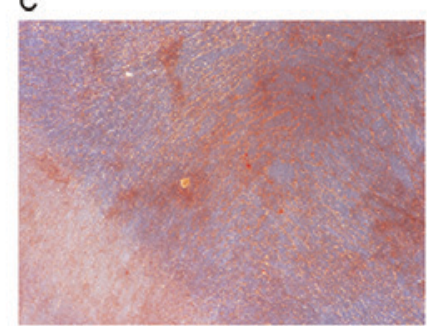

D

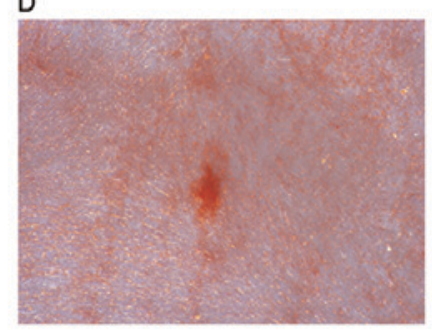

E

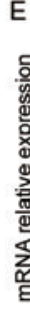

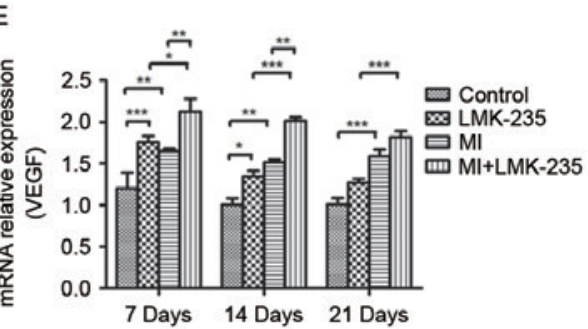

$\mathrm{F}$

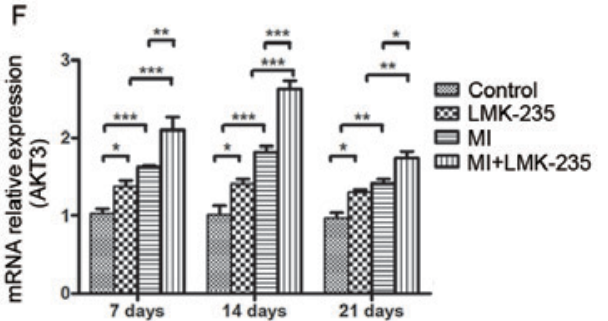

G

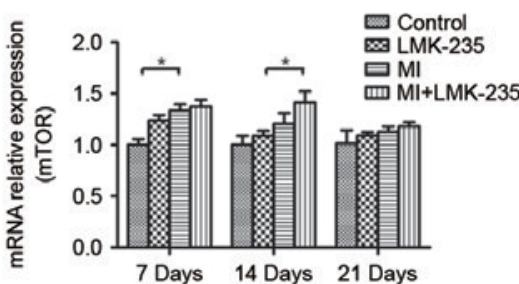

Figure 4. Alizarin Red S staining and the effect of the VEGF/AKT/mTOR pathway on odontoblast differentiation induced by LMK-235. Alizarin Red S staining of the (A) control, (B) LMK-235, (C) MI and (D) MI+LMK-235 groups (magnification, x100). mRNA expression levels of (E) VEGF, (F) AKT and (G) mTOR. Data are presented as the mean \pm standard deviation. ${ }^{*} \mathrm{P}<0.05,{ }^{* *} \mathrm{P}<0.01,{ }^{* * *} \mathrm{P}<0.001$. DSPP, dentin sialophosphoprotein; MI, mineralized inductive; VEGF, vascular endothelial growth factor; mTOR, mechanistic target of rapamycin; AKT, RAC-gamma serine/threonine-protein kinase.

The phenomenon mentioned above that DSPP expression was slightly higher in the MI+LMK-235 group compared with the MI group may be due to the overexpression of Runx2 at the late stage of DPSC odontoblast differentiation. The potential molecular mechanism of DSPP and Runx 2 expression underlying the role of LMK-235 needs to be further studied.

OCN, a gamma-carboxyglutamic acid containing protein, is always expressed during the late period of odontoblast and osteoblast differentiation (30). The expression of OCN mRNA demonstrated that OCN expression was not apparently altered by LMK-235, indicating that LMK-235 might promote odontoblast differentiation mainly at the early stage.

Alizarin Red S staining is regarded as a crucial method to detect odontoblast differentiation (31). More and larger calcified nodules and stronger staining was observed in the MI+LMK-235 group when compared with the MI group at day 21 , indicating that the formation of calcified nodules was increased by LMK-235.

The present study also aimed to illustrate the potential molecular mechanism of LMK-235 in odontoblast differentiation. In recent years, the phosphoinositide 3-kinase (PI3K)/AKT pathway has proven to serve a pivotal role in the growth and osteogenic differentiation of mesenchymal stem cells in vitro (32). AKT is regarded as the chief target of PI3K signalling. Other extracellular signals or growth factors, such as VEGF, might activate the PI3K/Akt signalling pathway to regulate fundamental cellular processes. VEGF silencing suppresses the proliferation of human SaOS-2 cells and reduces angiogenesis by inactivating the $\mathrm{VEGF} / \mathrm{PI} 3 \mathrm{~K} / \mathrm{AKT}$ pathway. Additionally, VEGF expression is regarded to be repressed by HDACs, especially HDAC4 (33). Moreover, an mTOR inhibitor attenuates osteoblast differentiation of human periodontal ligament cells and osteoblasts (34). Therefore, it was hypothesized that LMK-235 might promote odontoblast differentiation by activating he VEGF/AKT/mTOR pathway through reducing HDAC4 activity to a certain extent. The
RT-qPCR results indicated that VEGF and AKT mRNA expression in DPCs were increased markedly after odontoblast induction, and the promotion effects were enhanced by LMK-235. mTOR mRNA expression was slightly upregulated in the MI+LMK-235 group when compared with that of the MI group. This phenomenon might be caused by the fact that mTOR was partially activated, whereas some other pathways downstream might participate in the subsequent reaction of AKT activation. Thus, the VEGF/AKT/mTOR pathway might take part in odontoblast differentiation induced by LMK-235.

In conclusion, the present study demonstrated that a certain concentration of LMK-235, a specific HDAC4 and HDAC5 inhibitor, was proven to have the ability to promote DPC odontoblast differentiation without reducing cell proliferation in vitro, and the VEGF/AKT/mTOR signalling pathways might take part in this process. Depending on these results above, further studies on LMK-235 as a regulator of dental tissue regeneration are necessary, providing therapeutic application potential in the future.

\section{Acknowledgements}

The present study was supported the Medical Research Foundation of Guangdong Province (Guangzhou, China; grant no. A2016541), the President Foundation of Nanfang Hospital, Southern Medical University (Guangzhou, China; grant no. 2014C020), the Scientific Research Staring Foundation of Southern Medical University (Guangzhou, China; grant no. PY2014N051), and the National Natural Science Foundation of China (Beijing, China; grant no. 81371137).

\section{References}

1. Jo YY, Lee HJ, Kook SY, Choung HW, Park JY, Chung JH, Choung YH, Kim ES, Yang HC and Choung PH: Isolation and characterization of postnatal stem cells from human dental tissues. Tissue Eng 13: 767-773, 2007. 
2. Seo BM, Miura M, Gronthos S, Bartold PM, Batouli S, Brahim J, Young M, Robey PG, Wang CY and Shi S: Investigation of multipotent postnatal stem cells from human periodontal ligament. Lancet 364: 149-155, 2004.

3. Ji YM, Jeon SH, Park JY, Chung JH, Choung YH and Choung PH: Dental stem cell therapy with calcium hydroxide in dental pulp capping. Tissue Eng Part A 16: 1823-1833, 2010.

4. Arnsdorf EJ, Tummala P, Castillo AB, Zhang F and Jacobs CR: The epigenetic mechanism of mechanically induced osteogenic differentiation. J Biomech 43: 2881-2886, 2010.

5. Lehrmann H, Pritchard LL and Harel-Bellan A: Histone acetyltransferases and deacetylases in the control of cell proliferation and differentiation. Adv Cancer Res 86: 41-65, 2002.

6. Marks PA, Richon VM and Rifkind RA: Histone deacetylase inhibitors: Inducers of differentiation or apoptosis of transformed cells. J Natl Cancer Inst 92: 1210-1216, 2000.

7. Jeon EJ, Lee KY, Choi NS, Lee MH, Kim HN, Jin YH, Ryoo HM, Choi JY, Yoshida M, Nishino N, et al: Bone morphogenetic protein-2 stimulates Runx2 acetylation. J Biol Chem 281: 16502-16511, 2006.

8. Fu Y, Zhang P, Ge J, Cheng J, Dong W, Yuan H, Du Y, Yang M, Sun R and Jiang H: Histone deacetylase 8 suppresses osteogenic differentiation of bone marrow stromal cells by inhibiting histone H3K9 acetylation and RUNX2 activity. Int J Biochem Cell Biol 54: 68-77, 2014.

9. Huynh NC, Everts V, Pavasant P and Ampornaramveth RS Inhibition of histone deacetylases enhances the osteogenic differentiation of human periodontal ligament cells. J Cell Biochem 117: 1384-1395, 2016

10. Lee HW, Suh JH, Kim AY, Lee YS, Park SY and Kim JB: Histone deacetylase 1-mediated histone modification regulates osteoblast differentiation. Mol Endocrinol 20: 2432-2443, 2006.

11. Nissen-Meyer LS, Svalheim S, Taubøll E, Reppe S, Lekva T, Solberg LB, Melhus G, Reinholt FP, Gjerstad L and Jemtland R: Levetiracetam, phenytoin, and valproate act differently on rat bone mass, structure, and metabolism. Epilepsia 48: 1850-1860, 2007.

12. Paino F, La Noce M, Tirino V, Naddeo P, Desiderio V, Pirozzi G, De Rosa A, Laino L, Altucci L and Papaccio G: Histone deacetylase inhibition with valproic acid downregulates osteocalcin gene expression in human dental pulp stem cells and osteoblasts: Evidence for HDAC2 involvement. Stem Cells 32: 279-289, 2014.

13. Jin H, Park JY, Choi H and Choung PH: HDAC inhibitor trichostatin A promotes proliferation and odontoblast differentiation of human dental pulp stem cells. Tissue Eng Part A 19: 613-624, 2013.

14. Kwon A, Park HJ, Baek K, Lee HL, Park JC, Woo KM, Ryoo HM and Baek JH: Suberoylanilide hydroxamic acid enhances odontoblast differentiation. J Dent Res 91: 506-512, 2012.

15. Lu J, Qu S, Yao B, Xu Y, Jin Y, Shi K, Shui Y, Pan S, Chen L and Ma C: Osterix acetylation at K307 and K312 enhances its transcriptional activity and is required for osteoblast differentiation. Oncotarget 7: 37471-37486, 2016.

16. Xia ZY, Hu Y, Xie PL, Tang SY, Luo XH, Liao EY, Chen F and Xie H: Runx2/miR-3960/miR-2861 positive feedback loop is responsible for osteogenic transdifferentiation of vascular smooth muscle cells. Biomed Res Int 2015: 624037, 2015.

17. Hansen FK, Sumanadasa SD, Stenzel K, Duffy S, Meister S, Marek L, Schmetter R, Kuna K, Hamacher A, Mordmüller B, et al: Discovery of HDAC inhibitors with potent activity against multiple malaria parasite life cycle stages. Eur J Med Chem 82: 204-213, 2014

18. Li A, Liu Z, Li M, Zhou S, Xu Y, Xiao Y and Yang W: HDAC5, a potential therapeutic target and prognostic biomarker, promotes proliferation, invasion and migration in human breast cancer. Oncotarget 7: 37966-37978, 2016.
19. Livak KJ and Schmittgen TD: Analysis of relative gene expression data using real-time quantitative PCR and the 2(-Delta Delta C(T)) method. Methods 25: 402-408, 2001.

20. Ganai SA: Histone deacetylase inhibitor sulforaphane: The phytochemical with vibrant activity against prostate cancer. Biomed Pharmacother 81: 250-257, 2016.

21. Fu M, Shi W, Li Z and Liu H: Activation of mPTP-dependent mitochondrial apoptosis pathway by a novel pan HDAC inhibitor resminostat in hepatocellular carcinoma cells. Biochem Biophys Res Commun 477: 527-533, 2016.

22. Liu G, Xu G, Gao Z, Liu Z, Xu J, Wang J, Zhang C and Wang S: Demineralized dentin matrix induces odontoblastic differentiation of dental pulp stem cells. Cells Tissues Organs 201: 65-76, 2016.

23. Suzuki S, Sreenath T, Haruyama N, Honeycutt C, Terse A, Cho A, Kohler T, Müller R, Goldberg M and Kulkarni AB: Dentin sialoprotein and dentin phosphoprotein have distinct roles in dentin mineralization. Matrix Biol 28: 221-229, 2009.

24. Chen Y, Zhang Y, Ramachandran A and George A: DSPP is essential for normal development of the dental-craniofacial complex. J Dent Res 95: 302-310, 2016.

25. Gu S, Liang J, Wang J and Liu B: Histone acetylation regulates osteodifferentiation of human dental pulp stem cells via DSPP. Front Biosci (Landmark Ed) 18: 1072-1079, 2013.

26. Wang T, Liu H, Ning Y and Xu Q: The histone acetyltransferase p300 regulates the expression of pluripotency factors and odontogenic differentiation of human dental pulp cells. PLoS One 9: e102117, 2014.

27. Zhang X, Yang M, Lin L, Chen P, Ma KT, Zhou CY and Ao YF: Runx 2 overexpression enhances osteoblastic differentiation and mineralization in adipose-derived stem cells in vitro and in vivo. Calcif Tissue Int 79: 169-178, 2006.

28. Sun X, Wei L, Chen Q and Terek RM: HDAC4 represses vascular endothelial growth factor expression in chondrosarcoma by modulating RUNX2 activity. J Biol Chem 284: 21881-21890, 2009.

29. Li S, Kong H, Yao N, Yu Q, Wang P, Lin Y, Wang J, Kuang R, Zhao X, Xu J, et al: The role of runt-related transcription factor 2 (Runx2) in the late stage of odontoblast differentiation and dentin formation. Biochem Biophys Res Commun 410: 698-704, 2011.

30. Cutarelli A, Marini M, Tancredi V, D'Arcangelo G, Murdocca M, Frank C and Tarantino U: Adenosine Triphosphate stimulates differentiation and mineralization in human osteoblast-like Saos-2 cells. Dev Growth Differ 58: 400-408, 2016.

31. Tsukamoto Y, Fukutani S, Shin-Ike T, Kubota T, Sato S, Suzuki Y and Mori M: Mineralized nodule formation by cultures of human dental pulp-derived fibroblasts. Arch Oral Biol 37: 1045-1055, 1992.

32. Tong Y, Feng W, Wu Y,Lv H, Jia Y and Jiang D: Mechano-growth factor accelerates the proliferation and osteogenic differentiation of rabbit mesenchymal stem cells through the PI3K/AKT pathway. BMC Biochem 16: 1, 2015.

33. Dung TT, Yi YS, Heo J, Yang WS, Kim JH, Kim HG, Park JG, Yoo BC, Cho JY and Hong S: Critical role of protein L-isoaspartyl methyltransferase in basic fibroblast growth factor-mediated neuronal cell differentiation. BMB Rep 49: 437-442, 2016.

34. Bae WJ, Auh QS, Kim GT, Moon JH and Kim EC: Effects of sodium tri- and hexameta-phosphate in vitro osteoblastic differentiation in Periodontal Ligament and Osteoblasts, and in vivo bone regeneration. Differentiation 92: 257-269, 2016.

This work is licensed under a Creative Commons Attribution-NonCommercial-NoDerivatives 4.0 International (CC BY-NC-ND 4.0) License. 